\title{
Sentiment Analysis of Amazon Product Reviews Using Hybrid Rule-based Approach
}

\author{
Anjali Dadhich ${ }^{1}$ and Blessy Thankachan ${ }^{2}$ \\ ${ }^{1}$ Jaipur National University, Jaipur, Rajasthan, India \\ ${ }^{2}$ Jaipur National University, Jaipur, Rajasthan, India \\ Email: anjalidadhich01@gmail.com; blessyt218@gmail.com
}

Received: 26 October 2020; Accepted: 20 January 2021; Published: 08 April 2021

\begin{abstract}
In recent years, the retail market industry has taken a broad form to sell the products online and also to give the opportunity to customers to provide their valuable feedbacks, suggestions and recommendations. The opinion summarization and classification systems extract and identify a range of opinions about different online available products in a large text-based review set. This paper addresses and reviews the concepts of automatic identification of the sentiments expressed in the English text for Amazon and Flipkart products using Random Forest and K-Nearest Neighbor techniques. It presents a detailed comparative study of such existing sentiment analysis algorithms and methodologies on the basis of five key parameters. It results in evaluating their performance in terms of parameter usage and contributions. The paper also discusses their experimental results and challenges found. Therefore, this study shows the maximum usage of feature extraction, positive-negative sentiment, Amazon web source, mobile phone for a large set of reviews in the existing algorithms.
\end{abstract}

Index Terms: Sentiment Analysis, Random Forest, KNN, Opinion Summarization, Online Products.

\section{Introduction}

Automated sentiment analysis or opinion mining [1]-[3], [5]-[6], [8]-[17]is a prominent sub-field of the Natural Language Processing (NLP) and Machine Learning (ML), which interprets, extracts, summarizes and classifies the text [4], emotions and images [7] [18] in the subjective review data. It is also known as the subjective analysis to classify the text according to the polarity and orientation of the opinions. The sentiment polarity is expressed as bi-, tri- or multipolarity such as positive-negative, positive-neutral-negative, StrongPositive-Positive-Neutral-Negative-StrongNegative, respectively. The positive sentiment polarity occurs if the total number of positive word appearances is greater than the total number of negative word appearances. In the negative sentiment polarity, the total number of negative word appearances is greater than the total number of positive word appearances. The neutral sentiment polarity refers to the equal number of positive and negative word appearances.

SentiWordNet, a lexical resource, is used to provide three sentiment scores called positive, negative, and neutral in opinion mining. The objectivity is used as the opposite of subjectivity. Objectivity is not influenced by the personal opinions or feelings to represent the facts. Therefore, sentiment analysis is the contextual mining of text to identify and extract the subjective reviews in supply material. Furthermore it supports an enterprise to recognize the social sentiments in their brand, products or services while monitoring and managing online conversations and feedbacks [19] [20]. The performance measures in sentiment analysis are precision, recall and F1-measure. They are computed in terms of True Positive (TP), True Negative (TN), False Positives (FP) and False Negatives (FN), where TP, TN, FP and FN represent true classification as positive terms, truly classification as negative terms, false classification as positive terms and false classification as negative terms, respectively.

This paper aims to provide the detailed review and a systematic tour of the existing sentiment analysis and opinion mining methodologies using Random Forest (RF) and K-Nearest Neighbor (KNN) techniques. These algorithms are compared with each other on the basis of some key measuring parameters, which evaluate them for feature reduction, sentiment polarity, online web source, product types and review set size in English language only. The primary concern is to study the sentiment analysis of the online available product reviews of Amazon and Flipkart. Alongside, this paper illustrates the comparison among these existing algorithms in terms of their accuracy results and additional result findings. All these algorithms face many critical issues and challenges, which lead us towards the designing of a new, automated and efficient sentiment review analyzer.

$\mathrm{RF}$ is a collection of trees where each tree is different from each other. It is a supervised learning algorithm and is used in regression and classification problems. It constructs multiple Decision Trees (DT) and then it merges them 
together to get the absolute and stable values for review set training and testing. The reason behind the use of RF is that as it grows many classification trees so it resolves the instability problem of previous decision trees. To classify a new object from an input vector, this input can be run through every tree in the forest [6]. KNN is a simple algorithm which is used for both classification and regression problems. It stores all the available cases during training and then classifies new cases using a majority vote of its $\mathrm{k}$ neighbors.

This paper is organized as follows. This paper began with the introduction. Section 2 illustrates various concepts of sentiment analysis in terms of three components. The systematic tour and comparative study of various existing sentiment analysis and opinion classification approaches are described in Section 3. Section 4 depicts the accuracy results and additional findings on them. It also elaborated and compared various challenges, which were faced by each algorithm. Section 5 graphically evaluates the performance metric of these algorithms in terms of $\%$ usages. These results and observations are obtained by comparing these existing algorithms for some key parameters. Lastly, section 6 concludes the paper with the future scope.

\section{Sentiment Analysis Concepts}

Automatic sentiment analysis system is used to extract and classify the sentiments and emotions into different categories. The sentiment classification consists of the subjective part of an opinion, whereas emotion classification includes the projections or display of a feeling expressed in text. The sentiment analysis follows a sequence of steps such as the review collection, the text to lowercase conversion, punctuation and additional white space removal, tokenization, stop word removal, lemmatization, feature reduction and classification.

A few articles described the sentiment analysis and its related concepts as discussed next. Lior Goren Ruck, Senior Data Product Manager, Yotpo discussed about the customer feedback analysis with opinion mining for customer-driven brands in her article "How Customer Reviews Can Be Used for Opinion Mining" [19] on March 14, 2018. She addressed the concepts of opinion mining and customer reviews, the working of opinion mining and sentiment analysis, and topics and opinions extraction from the reviews. According to the recent article "Sentiment Analysis: Aspect-Based Opinion Mining - An investigation into sentiment analysis and topic modelling techniques" [20] in Towards Data Science, posted on October 27, 2020, Lowri Williams focused on the main challenges of growing user base with the large amount of data generated in natural language. She demonstrated the theoretical and implementation concepts in aspect-based sentiment analysis, which extracts both the entity described in the text of a product or service and the sentiment associated with these entities. With this she also discussed the topic modeling for text aspect extraction.

Figure 1 depicts the basic level of components in sentiment analysis, which are categorized as content based, source based and supplementary. The contents in the sentiment analysis process can include the text, images, and emotions, where the text can be monolingual, bi-lingual or N-lingual. The sentiment polarity is bi-polar, tri-polar or Npolar, such as positive-negative, positive-negative-neutral and many more.

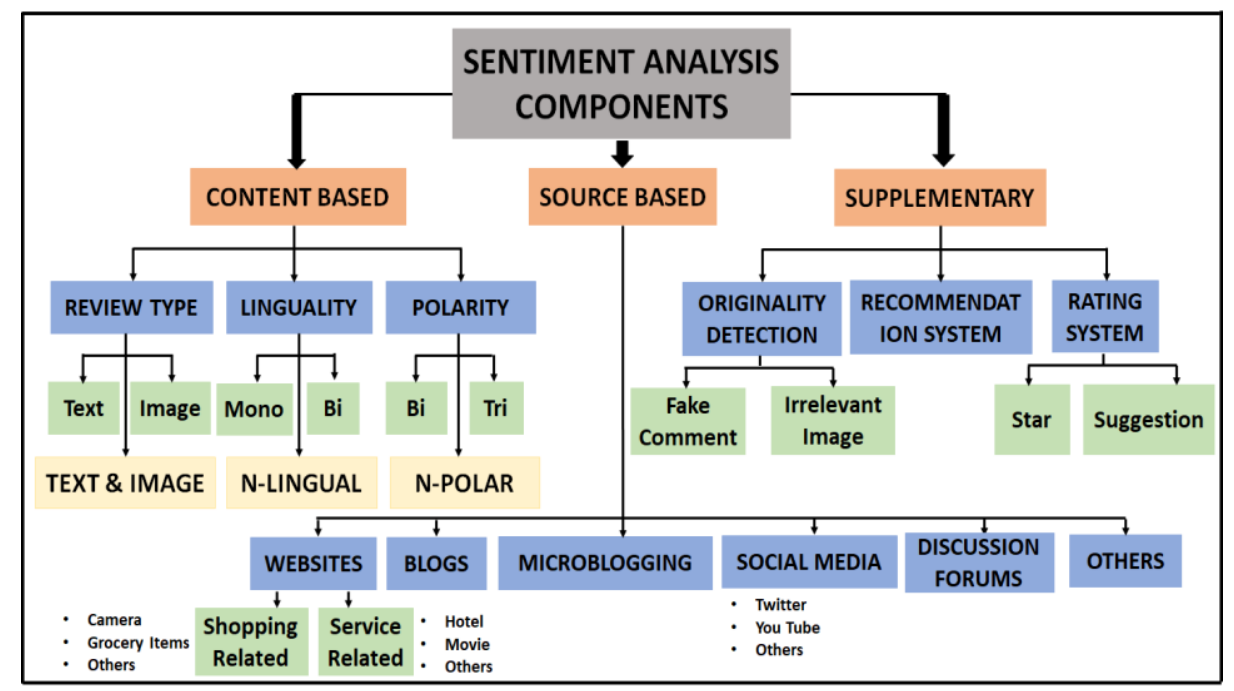

Fig. 1. Sentiment analysis and the components.

The online websites either sells the products or provides the services to the customers. Further the data sources in sentiment analysis are websites, blogs, microblogging, social media, discussion forums and other domains. The third component includes the concepts of originality detection of the reviews, recommendation system, and the star rating 
system. Many times, reviews are not straightforward, understandable and clear just like black and white, so the star rating system is used.

\section{Related Work}

This review illustrates the theoretical and algorithmicaspects of recent approaches in opinion summarization, classification and sentiment analysis using the RF and KNN techniques. Their observations and key findings are compared with each other with respect to six primary parameters which measure and evaluate their procedural concerns and practical results. The detailed review of these approaches is given below.

The sentiment expression extraction and sentiment classification approach [1] used the combination of four tree kernels, called Feature Sentiment Tree (FST), Generalized Feature-Sentiment Tree (GFST), and Boundary Marked GFST $\left(\mathrm{GFST}^{\mathrm{m}}\right)$ And Generalized Feature-Sentiment Tree with polarity labels $\left(\mathrm{GFST}^{\mathrm{p}}\right)$. It used three types of feature sets, such as lexicon features, syntactic features and semantic features for feature vector kernel, and then it represented each classification instance as a vector of n-dimensional predefined features. With this, it collected the reviews; split and indexed them into sentences using Indri2; and classified the sentiments using 10-fold cross validation. The automated product feature extraction system [2] used the combined approach of bootstrapping and ID3 along with Hu's method and Popescu's method. It followed the steps of data collection; preprocessing including extraction of consecutive noun sequences as candidate noun sequences and expression of the candidate noun sequences with classification features; feature extraction from candidate noun sequences; and lastly the final and new feature set extraction.

The review in [3] discussed the theoretical and practical concerns for efficient implementation of opinion classification in sentiment discovery using the classifiers such as Latent Dirichlet Allocation (LDA), Support Vector Machine (SVM), Multinomial Logistic Regression (MLR) or maximum entropy, Naïve Bayes (NB), Artificial Neural Network (ANN), DT, RF, and boosting and bagging algorithms with K-fold cross-validation. The empirical study and sentimental analysis method, presented in [5], classified the review text using NB by finding the review's subjectivity and objectivity; by correcting the spellings of the reviews using Word Net dictionary; by finding their semantic meanings; and lastly by calculating their polarity using DT. It followed the steps of review dataset collection; preprocessing including tokenization and stop word removal; feature selection; and then reviews classification and evaluation.

The sentiment analysis method [6] used a supervised lazy learning model along with syntactic rules for subjectivity/objectivity analysis and linguistic patterns identification. This method collected the reviews; pre-processed them with Part-of-Speech (POS) tagging through Penn Treebank annotation scheme with linguistic patterns identification through SentiWordNet; obtained the orientation of opinion sentences based on high score values of opinion words; extracted the product features and opinion words from subjective review sentences; extracted the opinions and summarized the reviews; and lastly classified them. Next sentiment analysis method [8] provided the optimized summary of text and image of customer reviews. Its steps include the data acquisition from input review corpus; the selection of some data from total data; the thread reading and loading into runtime memory; the input corpus size estimation after applying the pre-processing mechanisms; the stop word corpus acquisition including the word list for early elimination; the use of tokenization and stemming on their root words using the Porter stemming algorithm; the computation of token score and overall score; the determination of sentiment depth; the use of polar tag and emotion estimation; and finally the review classification.

The comparative study [9] presented the comparison between lexicon dictionary based approach with $\mathrm{N}$-grams and three ML algorithms. These ML algorithms were RF Learner with word vector and Principal Component Analysis (PCA) features, DT learner with document vector features, and RF with N-grams. The proposed method in [9] collected the data from corpus; divided it using EmEditor; cleaned and pre-processed reviews using punctuation eraser, stop word remover, case converter, and snowball stemming; obtained the features; and then classified the reviews. The sentiment analysis method [10] classified the customer reviews by data preprocessing; data resampling; feature extraction including dictionary based features and 50-d glove dictionary based features; and classification using classifiers such as Multinomial Naive Bayes (MNB), SVM with linear kernel, SVM with Radial Basis Function (RBF) kernel, KNN-4, 5, 6 and Long Short-Term Memory (LSTM) classifiers.

The sentiment analysis method [11] first performed the steps of data preprocessing including removal of stop words, punctuation marks, alpha-numeric character, HTML-tags, de-duplication, stemming and lemmatization; then text feature extraction using Term Frequency/Inverse Document Frequency (TF/IDF); then data splitting into training and testing; and finally the classification using KNN, LR and SVM with 5-fold cross validation.Next method [12] collected the data; computed the co-variance and correlation on it; selected the dependent and independent variables; pre-processed the data; applied the linear transformation, standardization, normalization and data mining; extracted the features; and finally classified them along with the detection of fake reviews.Another method [13] also began with the collection of online product reviews using web scraping, then it pre-processed it, extracted the features, and classified them.It replaced the output layer of the Convolution Neural Networks (CNN) by SVM.The method followed the steps 
of data collection, pre-processing, feature selection, detection process, and sentiment classification with K-fold cross validation[14].

The sentiment classification method of unlocked mobile reviews [15] predicted the product's customer rating based on its reviews and then classified these reviews using NB-SVM, RF, and MNB, gradient boosting, CNN, LSTM, and Stochastic Gradient Descent (SGD) classifiers. It included the steps of pre-processing including stop word removal, stemming, and POS tagging; level analysis with unigram classifiers; feature extraction; and lastly the classification.

The sentiment analysis [16] proposed a method Contextual Analysis (CA) to predict the performance of ML models, and to give an early warning to indicate when the performance of ML models were deteriorating with new datasets. This method collected the data from corpus; pre-processed it including tokenization, stop word removal, and normalisation with stemming and lemmatization; constructed a Hierarchy Knowledge Tree (HKT) with CA using unlabelled data; defined the threshold and created the branches for remaining words using the nodes created in a level of the HKT; and then classified the reviews. The multi-class classification [17] of comparative user reviews into different classes first acquired the dataset from YouTube using beautiful soup; pre-processed it including tokenization, POS tagging, spell correction, hash tag removal and other special characters; applied the multiple classifiers; and lastly evaluated the performance to recommend the best classifier.

In this way, this tour described the procedural details of many recent opinion and sentiment analysis based algorithms. Alongside, they show many differences with each other. Table 1 depicts such differences by comparing and evaluating these approaches on the basis of six measuring criteria, such as feature evaluation, sentiment polarity, dataset domain, product corpus, dataset size, and classification method.

Table 1.Comparison among existing proposals using discriminating criteria.

\begin{tabular}{|c|c|c|c|c|c|c|c|}
\hline $\begin{array}{l}\text { Ref. } \\
\text { No. }\end{array}$ & Problem Focused & $\begin{array}{l}\text { Feature } \\
\text { Evaluation } \\
\text { Method } \\
\end{array}$ & $\begin{array}{l}\text { Sentiment } \\
\text { Polarity }\end{array}$ & $\begin{array}{l}\text { Dataset } \\
\text { Domain }\end{array}$ & Product Corpus & Data set size & Classifier \\
\hline [1] & $\begin{array}{l}\text { Tree kernel based } \\
\text { opinion mining of } \\
\text { online product reviews }\end{array}$ & $\begin{array}{l}\text { Feature } \\
\text { Extraction }\end{array}$ & $\begin{array}{l}\text { Positive \& } \\
\text { Negative }\end{array}$ & $\begin{array}{l}\text { 1. Customer } \\
\text { review data } \\
\text { 2. Epinions }\end{array}$ & $\begin{array}{l}\text { 1. Digital camera, } \\
\text { cell phone, MP3, } \\
\text { DVD player, } \\
\text { router, antivirus } \\
\text { software } 2 . \text { No. } \\
\text { of sentences }\end{array}$ & $\begin{array}{l}\text { 1. Six products } \\
\text { with } 442 \text { reviews } \\
\text { 2. } 7,101,733 \\
\text { review bodies }\end{array}$ & $\begin{array}{lr}\text { SVM with tree } \\
\text { kernels } & \& \\
\text { polynomial kernel }\end{array}$ \\
\hline [2] & $\begin{array}{lr}\text { Product } & \text { feature } \\
\text { extraction using a } \\
\text { combined approach }\end{array}$ & $\begin{array}{l}\text { Feature } \\
\text { Extraction }\end{array}$ & $\begin{array}{l}\text { Positive \& } \\
\text { Negative }\end{array}$ & Amazon & Digital camera & $\begin{array}{l}\text { One product } \\
\text { with } 1000 \\
\text { reviews }\end{array}$ & - \\
\hline [3] & $\begin{array}{l}\text { Sentiment discovery } \\
\text { using classification } \\
\text { techniques }\end{array}$ & $\begin{array}{l}\text { Feature } \\
\text { Extraction }\end{array}$ & $\begin{array}{l}\text { Positive \& } \\
\text { Negative }\end{array}$ & $\begin{array}{l}\text { Amazon, } \\
\text { EBay \& } \\
\text { Twitter }\end{array}$ & $\begin{array}{l}\text { Samsung phone } \\
\text { \& others }\end{array}$ & $\begin{array}{ll}\text { Sufficient } & \\
\text { number } & \text { of } \\
\text { products } & \& \\
\text { reviews } & \end{array}$ & $\begin{array}{l}\text { LDA, SVM, LR, } \\
\text { NB, ANN, DT, } \\
\text { RF, boosting \& } \\
\text { bagging }\end{array}$ \\
\hline$[5]$ & \begin{tabular}{lr}
\multicolumn{2}{l}{ Sentimental } \\
using analysis \\
approach
\end{tabular} & $\begin{array}{l}\text { Feature } \\
\text { Selection }\end{array}$ & $\begin{array}{l}\text { Positive \& } \\
\text { Negative }\end{array}$ & Flipkart & $\begin{array}{l}\text { MOTO X Play } \\
\text { mobile phone }\end{array}$ & $\begin{array}{l}5 \text { products with } \\
10 \text { reviews }\end{array}$ & NB \& DT \\
\hline$[6]$ & $\begin{array}{l}\text { Summarization of } \\
\text { product reviews based } \\
\text { on features \&opinions }\end{array}$ & $\begin{array}{l}\text { Feature } \\
\text { Extraction }\end{array}$ & $\begin{array}{l}\text { Positive \& } \\
\text { Negative }\end{array}$ & Amazon & $\begin{array}{lr}\text { Canon digital } \\
\text { cameras } 1 \& 2, \\
\text { Nokia phone, } \\
\text { MP3 player \& } \\
\text { DVD player }\end{array}$ & $\begin{array}{l}5 \text { products } \& \\
\text { sufficient } \\
\text { number } \\
\text { reviews }\end{array}$ & KNN \\
\hline [8] & $\begin{array}{l}\text { Hierarchical sentiment } \\
\text { analysis for automatic } \\
\text { review classification }\end{array}$ & $\begin{array}{l}\text { Feature } \\
\text { Extraction }\end{array}$ & $\begin{array}{l}\text { Positive \& } \\
\text { Negative }\end{array}$ & $\begin{array}{l}\text { Flipkart, } \\
\text { Snapdeal\&B } \\
\text { estBuy }\end{array}$ & 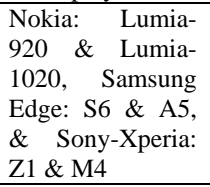 & $\begin{array}{l}6 \text { products } \& \\
\text { sufficient } \\
\text { number } \\
\text { reviews }\end{array}$ & RF \\
\hline [9] & $\begin{array}{l}\text { Study on opinion } \\
\text { mining on Amazon } \\
\text { product reviews }\end{array}$ & $\begin{array}{l}\text { Feature } \\
\text { Selection }\end{array}$ & $\begin{array}{l}\text { Positive \& } \\
\text { Negative }\end{array}$ & Amazon & Random selection & $\begin{array}{l}\text { Many products. } \\
57.3 \% \text { positive, } \\
25.56 \% \text { negative } \\
\& \quad 17.14 \% \\
\text { neutral review }\end{array}$ & $\begin{array}{l}\text { RF with word } \\
\text { vector \& N-gram, } \\
\& \text { DT with } \\
\text { document vector }\end{array}$ \\
\hline$[10]$ & $\begin{array}{l}\text { Sentiment } \\
\text { Amazon } \\
\text { reviews }\end{array}$ & $\begin{array}{l}\text { Feature } \\
\text { Extraction }\end{array}$ & $\begin{array}{l}\text { Positive \& } \\
\text { Negative }\end{array}$ & Amazon & $\begin{array}{l}\text { Random } \\
\text { Selection }\end{array}$ & 34627 reviews & $\begin{array}{l}\text { MNB, } \quad \text { SVM, } \\
\text { KNN \& LSTM }\end{array}$ \\
\hline [11] & $\begin{array}{l}\text { Sentiment } \\
\text { using ML }\end{array}$ & $\begin{array}{l}\text { Feature } \\
\text { Extraction }\end{array}$ & $\begin{array}{l}\text { Positive \& } \\
\text { Negative }\end{array}$ & $\begin{array}{l}\text { Amazon } \\
\text { (Kaggle) }\end{array}$ & $\begin{array}{l}\text { Food-based } \\
\text { products (Total } \\
74258 \text { products) }\end{array}$ & $\begin{array}{l}\text { Few } \\
\text { products.Sets of } \\
568454 \quad \& \\
100,000 \text { reviews } \\
\end{array}$ & KNN, LR \& SVM \\
\hline$[12]$ & $\begin{array}{l}\text { Sentiment analysis of } \\
\text { customer reviews on } \\
\text { social network. }\end{array}$ & $\begin{array}{l}\text { Feature } \\
\text { Extraction }\end{array}$ & $\begin{array}{l}\text { Positive \& } \\
\text { Negative }\end{array}$ & $\begin{array}{l}\text { Flipkart \& } \\
\text { Amazon }\end{array}$ & $\begin{array}{lr}\text { Mobile } & \text { phone } \\
\text { (Honor } & 7 \mathrm{~A}, \\
\text { Redimi } & 6, \\
\text { Realme } & 2, \\
\text { MotoX4 } & \text { Nokia } \\
6.1 \text { ) } & \\
\end{array}$ & $\begin{array}{l}\text { Five Products } \\
\text { with sufficient } \\
\text { number of } \\
\text { reviews }\end{array}$ & - \\
\hline
\end{tabular}




\begin{tabular}{|c|c|c|c|c|c|c|c|}
\hline [13] & $\begin{array}{lr}\text { Sentiment } & \text { analysis of } \\
\text { product } & \text { reviews } \\
\text { scraping } & \text { from web } \\
\text { pages } & \end{array}$ & $\begin{array}{l}\text { Feature } \\
\text { Extraction }\end{array}$ & $\begin{array}{l}\text { Positive, } \\
\text { Neutral \& } \\
\text { Negative }\end{array}$ & $\begin{array}{l}\text { Amazon, } \\
\text { Flipkart\&Sn } \\
\text { apdeal }\end{array}$ & $\begin{array}{l}\text { Random } \\
\text { Selection }\end{array}$ & $\begin{array}{l}\text { Forty-five } \\
\text { products \& \& } \\
43777 \text { reviews }\end{array}$ & $\begin{array}{lr}\text { KNN, SVM, RF, } \\
\text { CNN } \\
\text { CNN }\end{array}$ \\
\hline [14] & $\begin{array}{l}\text { Sentiment analysis of } \\
\text { social media network } \\
\text { using RF algorithm }\end{array}$ & $\begin{array}{l}\text { Feature } \\
\text { Selection }\end{array}$ & $\begin{array}{l}\text { Positive, } \\
\text { Neutral \& } \\
\text { Negative }\end{array}$ & Flipkart & $\begin{array}{l}\text { Clothes, toys, } \\
\text { tools \& hardware, } \\
\text { school supplies, } \\
\text { baby care, mobile } \\
\text { \& accessory }\end{array}$ & $\begin{array}{lr}\text { Six } & \text { products } \\
\text { with } & \text { good } \\
\text { number } & \text { of } \\
\text { reviews } & \end{array}$ & RF \& SVM \\
\hline [15] & $\begin{array}{lr}\text { Sentiment } & \text { analysis on } \\
\text { mobile phone reviews } \\
\text { using } \\
\text { learning }\end{array}$ & $\begin{array}{l}\text { Feature } \\
\text { Extraction }\end{array}$ & $\begin{array}{l}\text { Positive \& } \\
\text { Negative }\end{array}$ & $\begin{array}{l}\text { Amazon } \\
\text { (Kaggle) }\end{array}$ & $\begin{array}{l}\text { Unlocked mobile } \\
\text { phone }\end{array}$ & $\begin{array}{l}\text { One product } \\
\text { with more than } \\
413,840 \text { reviews }\end{array}$ & $\begin{array}{l}\text { Gradient } \\
\text { boosting, SGD, } \\
\text { MNB, LSTM, } \\
\text { RF, NB-SVM \& } \\
\text { CNN }\end{array}$ \\
\hline [16] & $\begin{array}{l}\text { Supervised ML } \\
\text { performance prediction } \\
\text { for sentiment analysis } \\
\text { using contextual } \\
\text { methods }\end{array}$ & $\begin{array}{l}\text { Feature } \\
\text { Extraction }\end{array}$ & $\begin{array}{l}\text { Positive \& } \\
\text { Negative }\end{array}$ & $\begin{array}{l}\text { Amazon } \\
\text { with four } \\
\text { different } \\
\text { domains }\end{array}$ & $\begin{array}{l}\text { Book, DVD, } \\
\text { kitchen, } \\
\text { electronics and } \\
\text { movie }\end{array}$ & $\begin{array}{l}\text { Five products \& } \\
13000 \text { reviews }\end{array}$ & $\begin{array}{lll}\text { MNB, } & \text { RF } \quad \& \\
\text { SVM } & & \end{array}$ \\
\hline [17] & $\begin{array}{l}\text { Sentiment } \\
\text { classification of user } \\
\text { reviews r using } \\
\text { supervised learning } \\
\text { with comparative } \\
\text { opinion mining }\end{array}$ & $\begin{array}{l}\text { Feature } \\
\text { Extraction }\end{array}$ & $\begin{array}{l}\text { Positive, } \\
\text { Negative \& } \\
\text { Neutral }\end{array}$ & You Tube & $\begin{array}{l}\text { Business-related } \\
\text { comparative } \\
\text { reviews on 1. iOS } \\
\text { vs Android } 2 . \\
\text { Microsoft } \\
\text { Google }\end{array}$ & $\begin{array}{l}\text { Three } \\
\text { products.1. } 6000 \\
\text { reviews 2. } 3000 \\
\text { reviews }\end{array}$ & $\begin{array}{l}\text { NB, SVM, RF, } \\
\text { KNN, DT, LR } \\
\text { \&gradient } \\
\text { boosting }\end{array}$ \\
\hline
\end{tabular}

In this way, it is seen that this study not only compared various opinion mining, sentiment analysis and feature based methodologies on the basis of six key parameters, but also elaborated their concepts and practical significance very clearly. Most of them worked upon the successful implementation of classification systems with a large set of product reviews, but still there is the need of an efficient sentiment analysis classification system for multiple online available products of multiple data domains and sources.

\section{Discussion on Results and Challenges}

Section 3 described and distinguished many algorithms of opinion classification and sentiment analysis. Their accuracy results, additional result findings, needs, challenges and limitations are compared with each other and are tabulated in Table 2. These systems evaluated their performance in terms of accuracy, precision, recall and F1-score. Many systems were implemented either with multiple classifiers or the hybrid classifier of two classifiers. Their results were also compared with each other and performance was evaluated. Further the future needs, challenges and limitations of these systems present the critical issues and scope of these existing algorithms.

Table 2. Comparison of accuracy results, additional result findings along with needs, challenges and limitations.

\begin{tabular}{|c|c|c|c|}
\hline $\begin{array}{l}\text { Ref. } \\
\text { No. }\end{array}$ & Accuracy & Additional results and findings & Needs, challenges and limitations \\
\hline [1] & $\begin{array}{l}89.56 \% \text { Accuracy, } \\
\text { Precision, 93.53\% } \\
\text { \&92.31\% F1-score }\end{array}$ & $\begin{array}{l}\text { Complexity reduction due to the use of tree } \\
\text { kernels. Best performance of GFST } \\
\& \mathrm{GFST}^{\mathrm{m}}+\text { Poly with } 14.51 \% \text {, } \mathrm{GFST}^{\mathrm{p}}+\mathrm{Poly} \\
\text { with } 15.79 \% \text { improvement over } \\
\text { baseline.Obtained better results with feature } \\
\text { vector kernel than traditional flat features. } \\
\text { Achieved best performance of tree kernels } \\
\text { with feature vector kernel. }\end{array}$ & $\begin{array}{l}\text { Challenge to design proper tree kernel spaces } \\
\text { for similarity evaluation. Worst performance } \\
\text { of opinion mining for negative opinions. } \\
\text { Issue of data sparsity. Impractical to use } \\
\text { syntactic structure features directly. No need } \\
\text { to extract each individual feature from each } \\
\text { parsing tree. }\end{array}$ \\
\hline$[2]$ & Recall: $81 \% \&$ Precision: $70 \%$ & $\begin{array}{l}\text { Used a combined method of bootstrapping \& } \\
\text { ID3 for feature extraction. }\end{array}$ & $\begin{array}{l}\text { Precision decreased due to increased recall. } \\
\text { Avoided textual pattern structure \& } \\
\text { similarity function of textual pattern. }\end{array}$ \\
\hline [3] & $\begin{array}{l}\text { Recall: } 0.0654 \text { in NB, } 0.8 \text { in } \\
\text { RF, } 0.6 \text { in LR, } 0.6 \text { in DT, } 0.8 \\
\text { in Bagging, \& } 0.2 \text { in SVM. }\end{array}$ & $\begin{array}{l}\text { Achieved highest recall accuracy with RF. } \\
\text { Found mean accuracy of cross validation: } \\
0.4625 \text { in DT, } 1 \text { in LR, } 0.875 \text { in SVM, \& } 0.825 \\
\text { in RF. }\end{array}$ & $\begin{array}{l}\text { Challenges were co-reference resolution, } \\
\text { sarcastic sentences, linguistic issues, opinion } \\
\text { spamming, \&volatility over time. }\end{array}$ \\
\hline [5] & High Accuracy. & $\begin{array}{l}\text { Better performance of Moto X Play (16 GB) } \\
\text { compared to Moto E \& Moto G. }\end{array}$ & $\begin{array}{l}\text { Need to improve the accuracy and system } \\
\text { performance. }\end{array}$ \\
\hline$[6]$ & $\begin{array}{lrr}\text { Average } & \text { recall: } & 0.967, \\
\text { precision: } & 0.761, \& & \text { F-Score: } \\
0.849 . & & \end{array}$ & Training accuracy was $81.37(+/-0.77 \%)$. & $\begin{array}{l}\text { Need to improve implicit features and } \\
\text { opinion strength. Got low precision values } \\
\text { than recall. Ignorance of some opinion } \\
\text { sentences and product features due to } \\
\text { grammatical errors in review sentences. }\end{array}$ \\
\hline$[8]$ & $\begin{array}{l}\text { Average accuracy: } 94.71 \% \\
\text { Accuracy: } 96.23 \% \text { without \& } \\
93.75 \% \quad \text { with manual } \\
\text { compression. Positivity: } \\
64.1 \% \text { in Samsung \& } 54.93 \% \\
\text { in Sony. }\end{array}$ & $\begin{array}{l}\text { Provided the image summary of reviews. } \\
\text { Found } 94.71 \% \text { Porter stemming accuracy. } \\
\text { Existing model outperformed the syntactic \& } \\
\text { algorithmic error categories. }\end{array}$ & $\begin{array}{l}\text { Need to improve the accuracy and system } \\
\text { performance. }\end{array}$ \\
\hline
\end{tabular}




\begin{tabular}{|c|c|c|c|}
\hline [9] & $\begin{array}{l}0.896 \text { (Best) using lexicon } \\
\text { dictionary based approach } \\
\text { with N-grams, \& } 0.5 \text { (worst) } \\
\text { using RF with word vector } \\
\text { and PCA. }\end{array}$ & $\begin{array}{l}\text { Achieved better performance with lexicon } \\
\text { based approach than other ML techniques. } \\
\text { Achieved accuracy of } 70 \% \text { in DT with } \\
\text { document vector features \&of } 85 \% \text { in RF with } \\
\text { N-grams. }\end{array}$ & $\begin{array}{l}\text { Used only } 500 \mathrm{MB} \text { data. Trained classifier } \\
\text { did not work with other domains. Failed to } \\
\text { predict reviews having spelling mistakes and } \\
\text { slangs. Failed to process ambiguous and } \\
\text { contradicting reviews. }\end{array}$ \\
\hline [10] & Good Accuracy. & $\begin{array}{l}\text { Average training accuracy: } 68.02 \% \text { \& testing } \\
\text { accuracy: } 65.6 \% \text {. Obtained best results with } \\
\text { LSTM. KNN-5 outperformed other two KNN } \\
\text { models. SVM with linear kernel slightly } \\
\text { outperformed SVM with RBF Kernel. Increase } \\
\text { in dictionary's length did not have too much } \\
\text { effect on accuracy }\end{array}$ & $\begin{array}{l}\text { Obtained worst result using glove mean than } \\
\text { normal word count due to weak individual } \\
\text { word feature set. Low accuracy due to data } \\
\text { imbalance. Did not find high no. of data } \\
\text { points from other resources. Had over fitting } \\
\text { problem in SVM with linear kernel. KNN } \\
\text { required much higher computation } \\
\text { complexity than NB \& SVM during training } \\
\text { time. Issue of curse of dimensionality. }\end{array}$ \\
\hline [11] & od Accu & - & $\begin{array}{l}\text { Need to maintain the balanced proportion of } \\
\text { positive and negative reviews. }\end{array}$ \\
\hline [12] & Good Accuracy. & ter \& negative & $\begin{array}{l}\text { Variation in customer rating for same } \\
\text { product. Problem of fake reviews. }\end{array}$ \\
\hline [13] & $\begin{array}{l}94.2 \% \text { precision, } 92.4 \% \\
\text { recall, 90.7\% F-score, \& } \\
92.4 \% \text { accuracy with Hybrid } \\
\text { SVM-CNN. }\end{array}$ & $\begin{array}{l}\text { Best performance with hybrid algorithm. Time } \\
\text { taken (milli-seconds): } 1400 \text { in KNN, } 1317 \text { in } \\
\text { SVM, } 1429 \text { in RF, } 1284 \text { in CNN, \& } 1102 \text { in } \\
\text { hybrid SVM-CNN. }\end{array}$ & $\begin{array}{l}\text { Need to improve the accuracy and system } \\
\text { performance. }\end{array}$ \\
\hline [14] & $\begin{array}{l}\text { 97\% accuracy (Best) with RF } \\
\& 92 \% \text { with SVM. }\end{array}$ & & $\begin{array}{l}\text { Need to improve the accuracy and system } \\
\text { performance. }\end{array}$ \\
\hline [15] & $\begin{array}{l}\text { Average accuracy: } \\
\text { precision }=72 \%, 85 \%, \\
\text { recall }=74.28 \%, \\
\text { measure }=69.14 \%\end{array}$ & $\begin{array}{l}\text { Obtained better prediction in RF than LSTM \& } \\
\text { CNN. Not found good results with gradient } \\
\text { boosting, MNB \& SGD. Maximum accuracy: } \\
85 \% \text { with RF. }\end{array}$ & $\begin{array}{l}\text { Need to improve the accuracy and system } \\
\text { performance. }\end{array}$ \\
\hline [16] & $\begin{array}{l}57 \% \text { to } 75 \% \text { prediction } \\
\text { accuracy with SVM. }\end{array}$ & $\begin{array}{l}\text { Estimated error within } 2.75 \text { to } 3.94 \& 2.30 \text { for } \\
3.51 \text { for average absolute differences. Lower } \\
\text { results of SVM than MNB \& RF. }\end{array}$ & $\begin{array}{l}\text { Need to improve the accuracy and system } \\
\text { performance. }\end{array}$ \\
\hline [17] & $\begin{array}{l}95 \% \quad \text { accuracy, } \quad 96 \% \\
\text { precision, 95\% recall \& 95\% } \\
\text { F-measure. }\end{array}$ & $\begin{array}{l}\text { Best result with RF in case } 1 \& \text { with DT in } \\
\text { case } 2 \text {. Also tested the system for Facebook vs } \\
\text { Twitter. }\end{array}$ & $\begin{array}{l}\text { Need to improve the accuracy and system } \\
\text { performance. }\end{array}$ \\
\hline
\end{tabular}

It can be seen that most of the existing algorithms and systems achieved high accuracy, precision, recall and F1score using single, multiple or hybrid classifiers. The hybrid classifiers achieved high performance results compared to the results of the single classifier. Additionally, these systems achieved and compared such results with a good set of online customer reviews for multiple online available products and services. Although these systems have implemented their algorithm with high level of efficiency and performance, yet they faced many critical issues and challenges.

These primary challenges include the need of improved algorithm; precision decrease with increased recall; curse of dimensionality; handling of fake, negative, ambiguous, contradicting and erroneous reviews; handling the large data set; issues in customer star rating and many others. Therefore, it can be stated that there is a high need of an efficient opinion classification system using multiple classifiers, which can result in very high performance with a large data set and can handle the problems of review types very effectively.

\section{Pcsa Design and Architecture}

The proposed Product Comment Summarizer and Analyzer (PCSA) system design is a generic, robust and fast system, which classifies online English comments collected from Flipkart shopping websites using five different supervised learning classification techniques. These techniques are Naïve Bayes, logistic regression, SentiWordNet, random forest and K-Nearest Neighbor. The PCSA system is designed in the training and testing phases.

During the system training, the user login registration form and credentials data base is set. It accepts multiples product URLs (Uniform Resource Locators) from Flipkart .It pre-processes the comments, segments the sentences, extracts their features, summarizes them or lastly classifies them using any one classification techniques. The system is trained with all five classification techniques. It is designed to classify the comments of multiple products through any one technique at a time. During the system testing, this proposed PCSA system is tested for a different unknown set of product comments collected from both websites. It goes through all these steps one by one and the chosen trained classifier categorizes these comments efficiently.

The entire PCSA model is designed using two stages called the training stage and the testing stage. With this, the system defines two storage media called Natural Language tool Kit based Corpus (NLTK based Corpus) and class repository. The NLTK based corpus includes the English dictionary, list of stop words, and WordNet information. The class repository includes three predefined classes called positive, negative and neutral. The PCSA system is trained on the known set of comments collected from Flipkart.

The trained classifiers classify the unknown set of comments. During the training stage, these comments are preprocessed for stop word removal, stemming and lemmatization. It makes use of the NLTK based corpus. It identifies the 
sentences on the basis of delimiters and segments the sentences. The system extracts all the important and relevant words, and it discards all the irrelevant words.

The system extracts the features from the important information. Further, these features train all the five classifiers. Then these classifiers perform the classification and classify all the comments into positive, negative and neutral comments. On the other side, all the comments are summarized and the system provides the rating to the each individual product in terms of the stars. The star rating uses the summarized results from all three classes and then provides the star rating to the chosen products of Amazon. During the testing stage, the system accepts the unknown set of comments. The system performs all the steps on this set of comments. Lastly, it chosen classifier classifies the comments into categories and summarizes all the class results to provide the start rating.

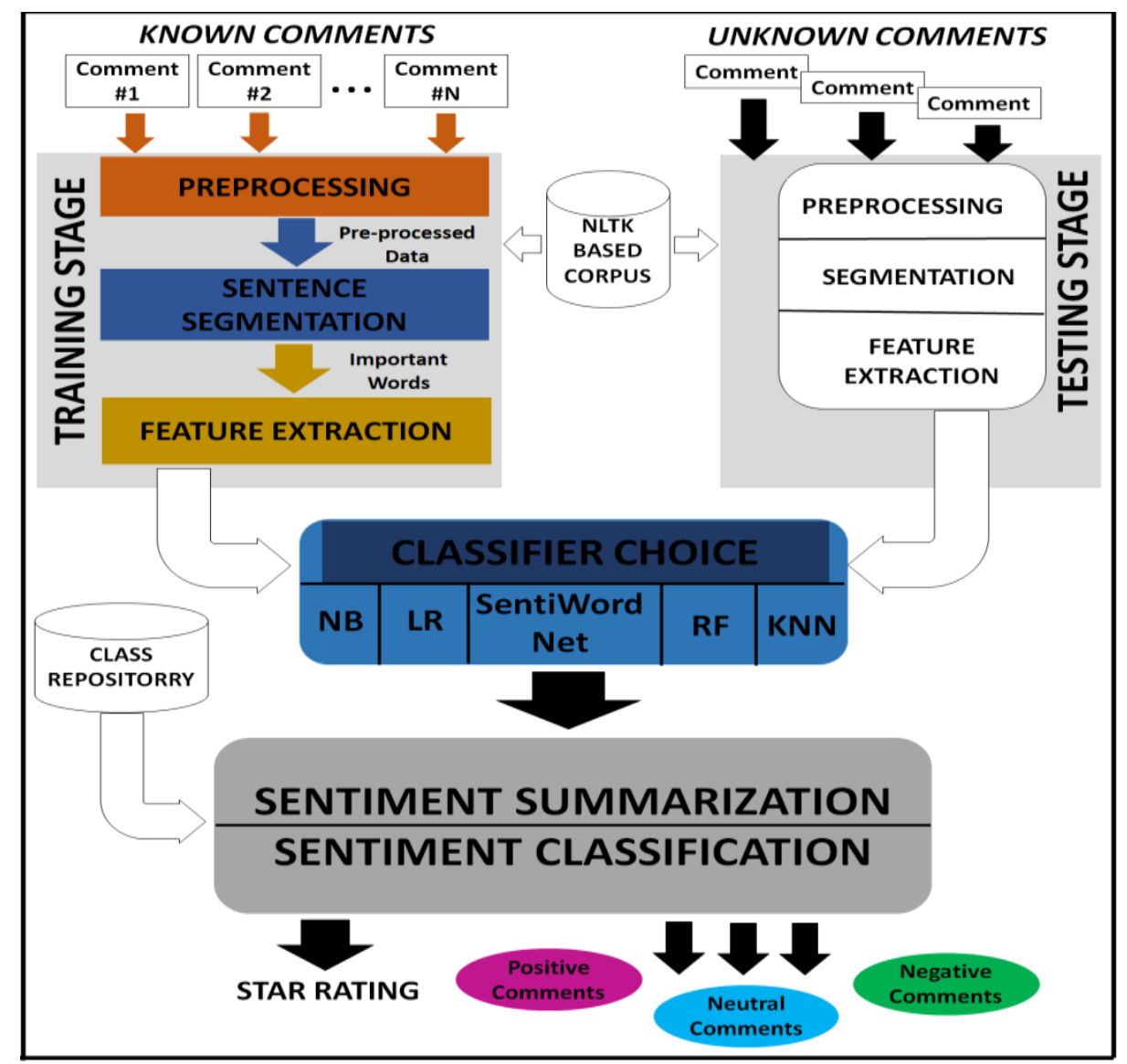

Fig.2. Detailed PCSA system Design

\subsection{System Description}

The proposed PCSA system follows a sequence of steps, and then summarizes and classifies the results efficiently. This section discusses the entire process of PCSA system in detail. These phases are user registration and login process, comment pre-processing, sentence segmentation feature extraction, training, comment summarization and classification.

\subsubsection{Registration and Login}

The system design has the homepage which is used to render the first page. It begins with the login details. If a user needs to get registered into the system and needs to create his/her own account. Then the registered user uses his/her login credentials to get access into it. Then the system asks the user to enter the number of URL links and the set of URL links of the online shopping website. In the browser, when a user enters a URL then it checks if the URL is present on the server side or not. If URL is not present, then the browser gives an error otherwise it redirects it for the comment collection. Therefore, the system gets both total number of links and product links in the array. It gets the number of links which the user wants to analyze, say, 1, 2, and 3 ... so on.

When the user writes the Flipkart website URLs, the system does the web crawling and finds the website. Further it shows the list of the products to the user which is available on the website. These websites include all types of products; say wearables, grocery items, household items etc. The user needs to select the products which should be processed by the system. 


\subsubsection{Comment Pre-Processing and Segmentation}

The system collects all the comments of the product from the webpage one-by-one. During the comment preprocessing, it pre-processes all the comments. These comments are pre-processed to get the important words and to discard the unimportant words. These unimportant words consume the space unnecessarily and increase the size of the database. For this it also uses NLTK based corpus. It segments all the sentences of the comments and retrieves only textual information from them. The system removes all the stop words, performs the stemming through the Porter stemmer, and does the lemmatization. The extracted important words are sent for the further processing. These words are the adjectives, nouns, adverbs and verbs.

\subsubsection{Feature Extraction and Training}

After performing the pre-processing and segmentation, the system extracts the features from the comments. The product features are battery life, resistance, looks etc. These features are used to train all five classifiers. During training, the data gets trained. The NB, LR, RF and KNN algorithms are simpler implementation than SentiWordNet algorithm. The structure of SentiWordNet algorithm is found complex and needs more efforts. The training data are made fit in the pipeline and pass it the CSV file of the comments method of the comment analyzer.

\subsubsection{Comment Summarization}

In the comment summarization, the system first gets the positive, negative and neutral reviews and then provides the summary of them. On the basis of these summarized reviews, the system is trained to provide the star rating for the particular product. The system functions read the CSV file created by the web scraper and predict the rating using pipeline. For this, it finds the average of the comments in each of its three categories. In this way, it gets the average of the total number of positive, negative and neutral product comments, and then finds the rating of the product.

This system also takes the actual star rating of the products from both websites. Each website provides the rating to each of its product available on its website, which is called the actual rating of the product. The proposed PCSA system provides the predicted rating to each product. Further, the PCSA system compares both actual and predicted ratings of the product.

\subsubsection{Comment Classification}

Along with the prediction process, the PCSA system categorizes the comments into positive, negative and neutral categories. It makes use of the class repository for this. This method also calculates the precision, recall and F1_score of the each chosen product. These three operations are performed using four variables true_positive, true_negative, false_positive, and false_negative.

After all this, all three values are stored in the class variables and are passed in the review scraper of Amazon depending upon the URL link. The review scraper stores all three values along with the average rating, title, image, and original rating. The system finds the product reviews for the selected website, discriminates the comments into three classes, finds the average rating and separates the rating predicted by the pipeline. Lastly, it performs the operations to get the precision, recall and F1_score results.

\section{Experimental Result and Discussion}

To perform these experiments, a large set of comments are collected for ten product categories from Amazon. Total three-fourth product sub-categories were used for training stage and one-fourth sub-categories were kept for testing stage. These results are obtained for categorical reviews, summarization and rating, and review classification.

Table 1.Training and testing data from Flipkart

\begin{tabular}{|c|c|c|c|}
\hline \multirow{2}{*}{ S.N. } & \multicolumn{3}{|c|}{ Flipkart } \\
\cline { 2 - 4 } & Training Reviews & Testing Reviews & $\begin{array}{c}\text { Total Number of } \\
\text { Reviews }\end{array}$ \\
\hline C-I & 12675 & 6210 & $\mathbf{1 8 8 8 5}$ \\
\hline C-II & 273 & 597 & $\mathbf{8 7 0}$ \\
\hline C-III & 256 & 3217 & $\mathbf{3 4 7 3}$ \\
\hline C-IV & 885 & 559 & $\mathbf{1 4 4 4}$ \\
\hline C-V & 13043 & 3718 & $\mathbf{1 6 7 6 1}$ \\
\hline C-VI & 1402 & 520 & $\mathbf{1 9 2 2}$ \\
\hline C-VII & 469 & 238 & $\mathbf{7 0 7}$ \\
\hline C-VIII & 16616 & 212 & $\mathbf{1 6 8 2 8}$ \\
\hline C-IX & 44 & 30 & $\mathbf{2 9 2 5}$ \\
\hline C-X & 2295 & 630 & $\mathbf{6 3 8 8 9}$ \\
\hline Total Reviews & $\mathbf{4 7 9 5 8}$ & $\mathbf{1 5 9 3 1}$ & \\
\hline
\end{tabular}


The review data set in PCSA system is collected for ten categories, such as mobile phones, cameras, laptops, Air Conditioners (AC), routers, televisions, books, kitchen items, furniture, and clothes and wearable items. These categories are called as C-I to C-X respectively. Each of categories has three sub-categories for training stage and one sub-category for testing stage. It depicts the total number of Flipkart training reviews for each sub-category along with its sub-category number, brand and model number.

In PCSA system, the categories used to test the system are Samsung Galaxy A50 (White, 64 GB) - 4 GB RAM, Canon PowerShot SX430 IS (20 MP, 45x Optical Zoom, 4x Digital Zoom, Black), Apple MacBook Air Core i5, LG 1.5 Ton 5 Star Split Dual Inverter AC, D-Link DIR-615 Wireless N 300 Router, Sony Bravia X7002G 108cm, ObjectOriented Programming with C++, Kitchen Burner- Sunflame - Smart Stainless Steel Manual Gas Stove, Table-WFH DeckUp Versa Engineered Wood, and HONOR Band 5 Watch. The reviews for these ten categories were collected and called C-I to C-X respectively.

Table 2. Positive, negative and neutral reviews for Flipkart

\begin{tabular}{|c|c|c|c|c|c|}
\hline \multirow[b]{2}{*}{ S.N. } & \multirow{2}{*}{$\begin{array}{l}\text { Category } \\
\text { Number }\end{array}$} & \multicolumn{4}{|c|}{ Flipkart Polarity } \\
\hline & & +ve Reviews & -ve Reviews & $\begin{array}{l}\text { Neutral } \\
\text { Reviews }\end{array}$ & $\begin{array}{c}\text { Total } \\
\text { Reviews }\end{array}$ \\
\hline 1 & C-I & Positive Reviews & $\begin{array}{l}\text { Negative } \\
\text { Reviews }\end{array}$ & $\begin{array}{l}\text { Neutral } \\
\text { Reviews }\end{array}$ & $\begin{array}{c}\text { Total } \\
\text { Reviews }\end{array}$ \\
\hline 2 & $\mathrm{C}$-II & 28204 & 2679 & 167 & 31050 \\
\hline 3 & C-III & 2528 & 398 & 59 & 2985 \\
\hline 4 & C-IV & 13683 & 2318 & 84 & 16085 \\
\hline 5 & $\mathrm{C}-\mathrm{V}$ & 2339 & 404 & 52 & 2795 \\
\hline 6 & $\mathrm{C}-\mathrm{VI}$ & 16664 & 1755 & 171 & 18590 \\
\hline 7 & C-VII & 2410 & 166 & 24 & 2600 \\
\hline 8 & C-VIII & 950 & 214 & 26 & 1190 \\
\hline 9 & C-IX & 803 & 233 & 24 & 1060 \\
\hline 10 & $\mathrm{C}-\mathrm{X}$ & 117 & 29 & 4 & 150 \\
\hline \multicolumn{2}{|c|}{ Total Polarity } & 3008 & 133 & 9 & 3150 \\
\hline & & 70706 & 8329 & 620 & 79655 \\
\hline
\end{tabular}

Table 3. Product rating by PCSA and Flipkart along with the rating difference.

\begin{tabular}{|c|c|c|c|c|c|c|c|c|c|}
\hline \multirow[b]{2}{*}{ S.N. } & \multirow[b]{2}{*}{ Category Number } & \multicolumn{8}{|c|}{ PCSA System Predicted Rating } \\
\hline & & NB & LR & SentiWordNet & $\mathbf{R F}$ & KNN & $\begin{array}{c}\text { Average } \\
\text { Rating }\end{array}$ & $\begin{array}{c}\text { Actual } \\
\text { Rating } \\
\text { from } \\
\text { Flipkart }\end{array}$ & $\begin{array}{c}\text { Rating } \\
\text { Difference }\end{array}$ \\
\hline 1 & C-I & 5 & 5 & 5 & 5 & 5 & 5 & 4.3 & -0.7 \\
\hline 2 & C-II & 4.9 & 5 & 4.8 & 5 & 5 & 4.94 & 4 & -0.94 \\
\hline 3 & C-III & 4.1 & 4.2 & 4.1 & 4.1 & 4.5 & 4.2 & 4.2 & $\mathbf{0}$ \\
\hline 4 & C-IV & 4 & 4.1 & 4 & 4 & 4.2 & 4.06 & 4 & -0.06 \\
\hline 5 & $\mathrm{C}-\mathrm{V}$ & 3.9 & 4 & 3.9 & 4.1 & 4 & 3.98 & 3.8 & -0.18 \\
\hline 6 & $\mathrm{C}-\mathrm{VI}$ & 4.8 & 4.9 & 4.8 & 5 & 4.9 & 4.88 & 4.3 & -0.58 \\
\hline 7 & C-VII & 4.2 & 4.5 & 4.6 & 4.5 & 4 & 4.36 & 4.2 & -0.16 \\
\hline 8 & C-VIII & 4.1 & 4.1 & 4.3 & 4.4 & 3.8 & 4.14 & 3.9 & -0.24 \\
\hline 9 & C-IX & 4.5 & 4.5 & 4.4 & 4.4 & 4.5 & 4.46 & 5 & 0.54 \\
\hline 10 & $\mathrm{C}-\mathrm{X}$ & 4.8 & 4.8 & 5 & 4.4 & 4.4 & 4.68 & 4.2 & -0.48 \\
\hline & Final Total & 4.43 & 4.51 & 4.49 & 4.49 & 4.43 & 4.47 & 4.19 & -0.28 \\
\hline
\end{tabular}

\subsection{Results on Review Classification}

The PCSA system performed the comment classification very efficiently and achieved high accuracy with overall testing dataset. This accuracy is computed by averaging the results of precision, recall and F1-Score.Table 4 depict the system classification results in terms of precision, recall and F1-Score for Amazon products, respectively. They also show the final total of precision, recall, F1-score and accuracy for Amazon. The PCSA system achieved total 0.942 precision, 0.8816 recall, $0.9104 \mathrm{~F} 1$-score and 0.91133 accuracy with Flipkart. 
Table 4. Precision, recall and F1-Score results with all classifiers for Flipkart products.

\begin{tabular}{|c|c|c|c|c|c|c|c|c|c|c|c|c|c|c|c|c|c|c|c|}
\hline \multirow[b]{2}{*}{$\begin{array}{l}\text { Category } \\
\text { Number }\end{array}$} & \multicolumn{3}{|c|}{ Naïve Bayes } & \multicolumn{3}{|c|}{ Logistic Regression } & \multicolumn{3}{|c|}{ SentiWordNet } & \multicolumn{3}{|c|}{ Random Forest } & \multicolumn{3}{|c|}{ K-Nearest Neighbor } & \multirow[b]{2}{*}{$\begin{array}{c}\text { Total } \\
\text { Precision }\end{array}$} & \multirow[b]{2}{*}{$\begin{array}{l}\text { Total } \\
\text { Recall }\end{array}$} & \multirow[b]{2}{*}{$\begin{array}{l}\text { Total F1- } \\
\text { Measure }\end{array}$} & \multirow[b]{2}{*}{$\begin{array}{c}\text { Total } \\
\text { Accuracy }\end{array}$} \\
\hline & Precision & Recall & \begin{tabular}{c|} 
F1- \\
Measure
\end{tabular} & Precision & Recall & \begin{tabular}{c|} 
F1- \\
Measure
\end{tabular} & Precision & Recall & \begin{tabular}{c|} 
F1- \\
Measure
\end{tabular} & Precision & Recall & $\begin{array}{c}\text { F1- } \\
\text { Measure }\end{array}$ & Precision & Recall & $\begin{array}{c}\text { F1- } \\
\text { Measure }\end{array}$ & & & & \\
\hline C-I & 0.83 & 0.71 & 0.77 & 0.72 & 0.76 & 0.74 & 0.83 & 0.52 & 0.64 & 0.78 & 0.74 & 0.76 & 0.72 & 0.59 & 0.65 & 0.776 & 0.664 & 0.712 & 0.71733 \\
\hline C-II & 0.96 & 0.78 & 0.86 & 0.92 & 0.86 & 0.89 & 0.96 & 0.81 & 0.88 & 0.96 & 0.86 & 0.91 & 0.96 & 0.83 & 0.89 & 0.952 & 0.828 & 0.886 & 0.88867 \\
\hline C-III & 0.91 & 0.88 & 0.9 & 0.9 & 0.87 & 0.88 & 0.88 & 0.65 & 0.75 & 0.9 & 0.92 & 0.91 & 0.82 & 0.74 & 0.78 & 0.882 & 0.812 & .844 & 0.846 \\
\hline C-IV & 0.98 & 1 & 1 & 1 & 0.98 & 0.97 & 1 & 1 & 1 & 0.96 & 0.98 & 1 & 1 & 0.97 & 1 & 0.988 & 0.986 & 0.994 & 0.98933 \\
\hline C-V & 0.95 & 0.95 & 0.95 & 0.95 & 0.95 & 0.95 & 0.95 & 1 & 0.97 & 0.84 & 0.94 & 0.89 & 1 & 0.96 & 1 & 0.938 & 0.96 & 0.952 & 0.95 \\
\hline C-VI & 1 & 0.9 & 0.95 & 1 & 0.9 & 0.95 & 0.94 & 0.89 & 0.92 & 1 & 0.9 & 0.95 & 0.9 & 0.98 & 0.95 & 0.968 & 0.914 & 0.944 & 0.942 \\
\hline C-VII & 1 & 0.85 & 0.92 & 1 & 0.79 & 0.88 & 0.91 & 0.77 & 0.83 & 0.91 & 0.83 & 0.87 & 1 & 0.79 & 0.88 & 0.964 & 0.806 & 0.876 & 0.882 \\
\hline C-VIII & 1 & 1 & 1 & 1 & 0.73 & 0.85 & 1 & 1 & 1 & 1 & 1 & 1 & 1 & 1 & 1 & 1 & 0.946 & 0.97 & 0.972 \\
\hline C-IX & 1 & 0.93 & 0.97 & 1 & 0.93 & 0.97 & 0.93 & 0.93 & 0.93 & 0.93 & 0.93 & 0.93 & 1 & 0.93 & 0.97 & 0.972 & 0.93 & 0.954 & 0.952 \\
\hline$c-x$ & 1 & 0.95 & 0.97 & 1 & 0.95 & 0.97 & 1 & 1 & 1 & 1 & 0.95 & 0.97 & 0.9 & 1 & 0.95 & 0.98 & 0.97 & 0.972 & 0.974 \\
\hline Total & 0.963 & 0.895 & 0.929 & 0.949 & 0.872 & 0.905 & 0.94 & 0.857 & 0.892 & 0.928 & 0.905 & 0.919 & 0.93 & 0.879 & 0.907 & 0.942 & 0.8816 & 0.9104 & 0.91133 \\
\hline
\end{tabular}

\section{Evaluation of Performance Metrics}

The section 3 illustrated the detailed review and comparative analysis of many existing sentiment analysis and opinion classification algorithms for recent years. Such comparison discriminated their methodologies on the basis of five primary parameters, such as, feature reduction, sentiment polarity and orientation, data domains and sources, product types and classifiers. Their performance is measured and analyzed on the basis of these parameters and is shown graphically through subsections 5.1 to 5.4.

\subsection{Based on feature evaluation and polarity}

Figure 3 depicts the $\%$ usage of the various feature reduction techniques and the sentiment polarity and orientation. On one side, it is observed that most of the research works implemented the feature extraction method in their sentiment analysis model as their most preferred method. On the other side, these works designed bi-polar sentiment analysis systems as their primary choice. In both the cases, they contributed $80 \%$ with the feature extraction and also $80 \%$ with the positive and negative reviews. The second and remaining $20 \%$ contribution include the feature selection method in reduction techniques and the positive, negative and neutral review category in sentiment polarity.

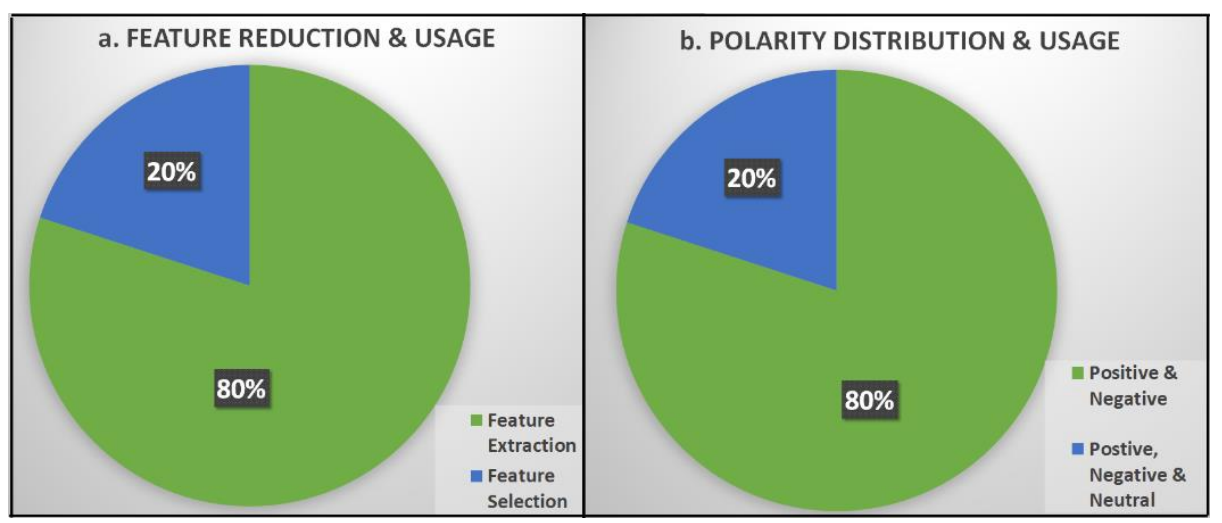

Fig. 3.\% Usage of a. feature reduction techniques b. sentiment polarity categories in existing systems.

\subsection{Based on data domain and sources}

Figure 4 depicts the $\%$ usage of various data sources and domains in existing systems which were used to collect the different online product reviews and data sets. These systems worked with the online data sources and websites such as Amazon, Flipkart, Snapdeal, Epinions, Ebay, Twitter, Bestbuy, You Tube, and Customer review data. It is observed that most of these contributors used the Amazon, with $66.67 \%$ usage, as their primary source of review collection for online products. The range of $\%$ contributions varies from $6.67 \%$ to $33.33 \%$ for other sources and websites. 


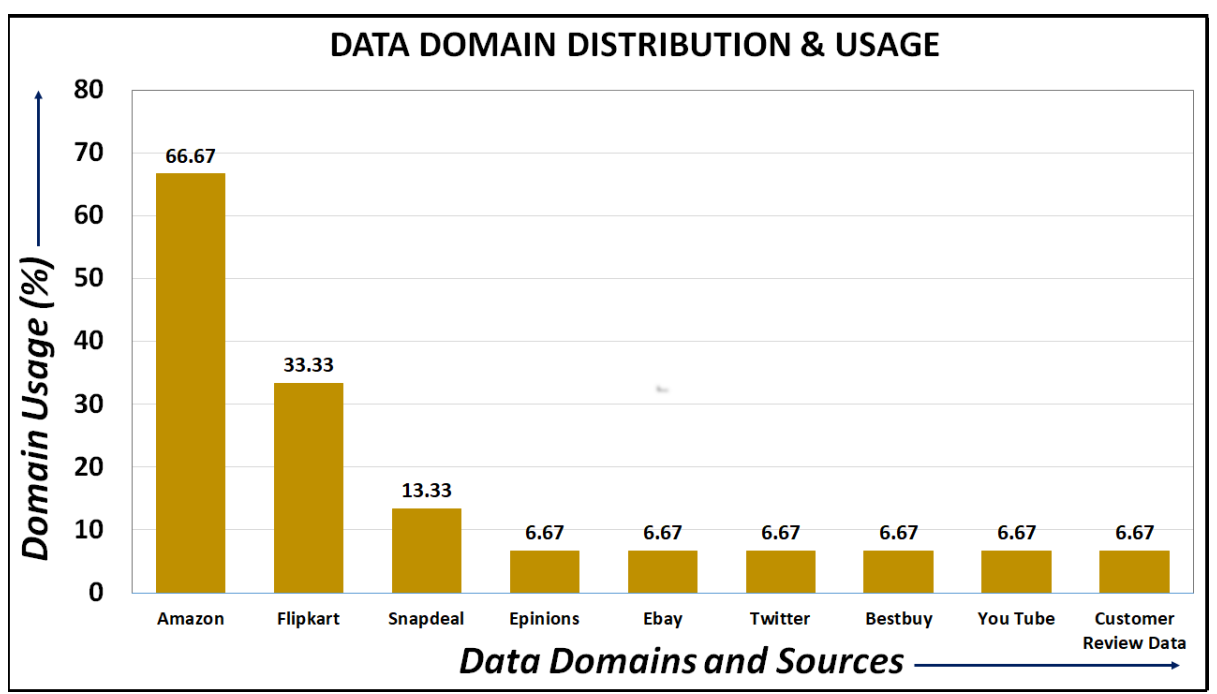

Fig. 4. \% Usage of data domains and sources in existing systems.

\subsection{Based on product type}

Figure 5 depicts the $\%$ usage of various types of products under different data sources, domains and websites for the existing systems. These systems found the results using many products such as Mobile phone, DVD player, camera, MP3 player, router, antivirus, food items, movie, book, business related, sentence based, other electronics, and clothes \& wearable's. It is observed that most of these contributors used the product, mobile phone, with $53.33 \%$ usage, as their first choice. The range of $\%$ contributions for other products varies from $20 \%$ to $6.67 \%$ as shown in Figure 5.

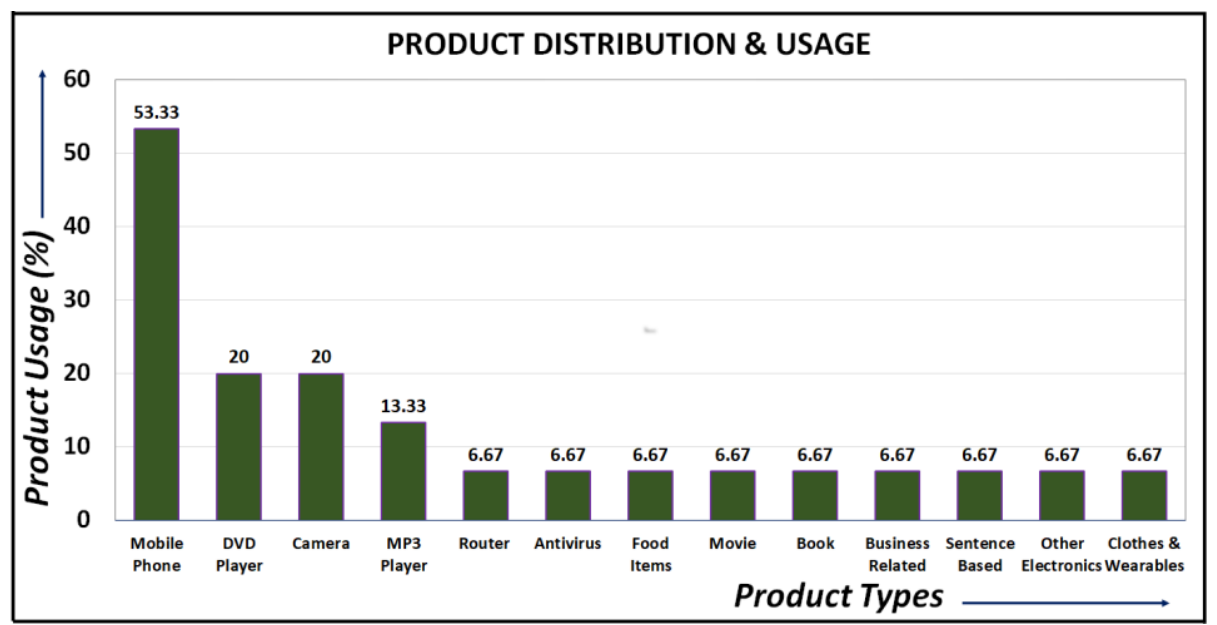

Fig. 5.\% Usage of online available products in various sources.

\subsection{Based on classifier used}

Figure 6 depicts the \% usage of various classifiers in the existing systems. These systems implemented their algorithms with many different single and hybrid classifiers such as SVM, RF, NB, ANN, DT, KNN, LR, gradient boosting, LDA, bagging, SVM-CNN, SGD and NB-SVM. It is observed that most of these contributors used SVM and RF classifiers, with 53.33\% usage, as their first choice. The range of \% contributions of other classifiers vary from $6.67 \%$ to $40 \%$ as shown in Figure 6. 


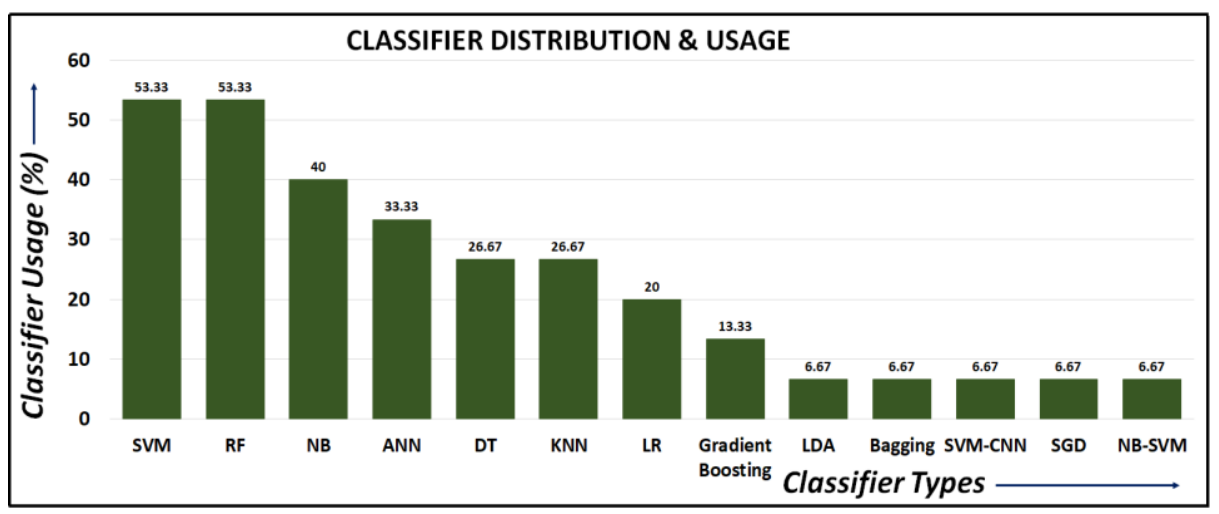

Fig. 6.\% Usage of different classifiers involved in existing systems.

\section{Conclusion and Future Scope}

The proposed PCSA system summarized and classified the comments obtained from the data domains of Amazon. The system polarity was checked for positive, negative and neutral comments using supervised learning algorithms such as NB, LR, SentiWordNet, RF and KNN techniques. This system found the final inclination towards the positive comments. The detailed review and survey provided the strong base towards the need of the PCSA system using multiple classifiers. Future scope will be implementation of this system using the hybrid and multiple supervised learning techniques.

\section{References}

[1] Jiang, P., Zhang, C., Fu, H., Niu, Z., Yang, Q: An approach based on tree kernels for opinion mining of online product reviews. In: IEEE International Conference on Data Mining, pp. 256-265. IEEE Press (2010).

[2] Li, Z.: Product feature extraction with a combined approach. In: Third International Symposium on Intelligent Information Technology and Security Informatics, pp. 686-690. IEEE Press (2010).

[3] Adinarayana, S., Ilavarasan, E.: Classification techniques for sentiment discovery-a review. In: International Conference on Signal Processing, Communication, Power and Embedded System, pp. 396-400. IEEE Press (2016).

[4] Puri, S., Kaushik, S.: An enhanced fuzzy similarity based concept mining model for text classification using feature clustering. In: Students' Conference on Engineering and Systems, pp. 1-6. IEEE Press (2012).

[5] Kaur, G., Singla, A.: Sentimental analysis of Flipkart reviews using Naïve Bayes and decision tree algorithm. International Journal of Advanced Research in Computer Engineering \& Technology 5(1), 149-153 (2016).

[6] Khan, J., Jeong, B. S.: Summarizing customer review based on product feature and opinion. In: Proceedings of the 2016 International Conference on Machine Learning and Cybernetics, pp. 158-165. IEEE Press (2016).

[7] Puri, S., Singh, S. P.: A technical study and analysis of text classification techniques in N-lingual documents. In: International Conference on Computer Communication and Informatics, pp. 1-6. IEEE Press (2016).

[8] Kaur, J., Bansal, M.: Hierarchical sentiment analysis model for automatic review classification for E-commerce users. In: Banati H., Bhattacharyya S., Mani A., Köppen M. (eds) Hybrid Intelligence for Social Networks, pp. 249-267. Springer, Cham (2017).

[9] Ejaz, A., Turabee, Z., Rahim, M., Khoja, S.: Opinion mining approaches on Amazon product reviews: a comparative study. In: International Conference on Information and Communication Technologies, pp. 173-179. IEEE Press (2017).

[10] Tan, W., Wang, X., Xu, X.: Sentiment analysis for Amazon reviews. In: International Conference, pp. 1-5. (2018).

[11] Diwakar, D., Kumar, R., Gour, B., Khan, A. U.: Proposed machine learning classifier algorithm for sentiment analysis. In: Sixteenth International Conference on Wireless and Optical Communication Networks. IEEE Press (2019).

[12] Sumedha, Johari, R.: SARPS: Sentiment analysis of review(s) posted on social network. In: Singh M., Gupta P., Tyagi V., Flusser J., Ören T., Kashyap R. (eds) Advances in Computing and Data Sciences. Communications in Computer and Information Science, vol. 1045, pp. 326-337. Springer, Singapore (2019).

[13] Suganya, E., Vijayarani, S.: Sentiment analysis for scraping of product reviews from multiple web pages using machine learning algorithms. In: Abraham A., Cherukuri A., Melin P., Gandhi N. (eds) Intelligent Systems Design and Applications. Advances in Intelligent Systems and Computing, vol. 941, pp. 677-685. Springer, Cham (2019).

[14] Karthika, P., Murugeswari, R., Manoranjithem, R.: Sentiment analysis of social media network using random forest algorithm. In: International Conference on Intelligent Techniques in Control, Optimization and Signal Processing, pp. 1-5. IEEE Press (2019).

[15] Shaheen, M., Awan, S. M., Hussain, N., Gondal, Z. A.: Sentiment analysis on mobile phone reviews using supervised learning techniques. International Journal of Modern Education and Computer Science 7, 32-43 (2019).

[16] Aziz, A. A., Starkey, A.: Predicting supervise machine learning performances for sentiment analysis using contextual-based approaches. IEEE Access 8, 17722-17733(2020). 
[17] Khan, A. et. al.: Sentiment classification of user reviews using supervised learning techniques with comparative opinion mining perspective. In: Arai K., Kapoor S. (eds) Advances in Computer Vision. Advances in Intelligent Systems and Computing, vol. 944, pp. 23-29. Springer, Cham (2020).

[18] Puri, S., Singh, S. P.: Advanced applications on bilingual document analysis and processing systems. International Journal of Applied Metaheuristic Computing 11(4), 149-193 (2020).

[19] YOTPO Blog, https://www.yotpo.com/blog/opinion-mining/, last accessed 2020/12/1.

[20] Towards Data Science, https://towardsdatascience.com/\%EF\%B8\%8F-sentiment-analysis-aspect-based-opinion-mining$72 \mathrm{a} 75 \mathrm{e} 8 \mathrm{c} 8 \mathrm{a} 6 \mathrm{~d}$, last acc0

\section{Authors' Profiles}

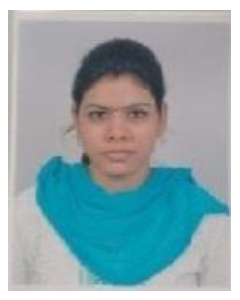

Anjali Dadhich received her master's degree in Computer Science from Banasthali Vidhyapeeth, Jaipur, India in 2014 and bachelor's degree from Mewar Girls College, Rajasthan, India in 2011. She is doing Ph.D. from JNU, Jaipur, and Rajasthan in the department of computer science. she published 10 papers in various international journals attended many conferences. She has very much interest to do research on Data mining and NLP.

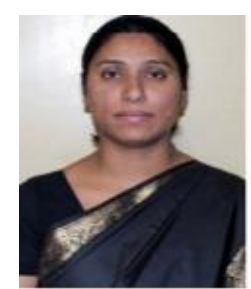

Dr. Blessy Thankachan, received her Ph.D. in the field of Software engineering, University of Rajasthan, Jaipur, India. Currently she is Assistant Director in Jaipur National University, India. Her areas of specialization include Software engineering and optimization. She has published many research articles in International and National Journals and Conferences.

How to cite this paper: Anjali Dadhich, Blessy Thankachan, " Sentiment Analysis of Amazon Product Reviews Using Hybrid Rulebased Approach ", International Journal of Engineering and Manufacturing (IJEM), Vol.11, No.2, pp. 40-52, 2021. DOI: 10.5815/ijem.2021.02.04 